\title{
Allergic Contact Dermatitis in Atopic Dermatitis
}

\section{Suwimon Pootongkam, MD Susan Nedorost, $M D^{*}$}

\author{
Address \\ *Department of Dermatology, University Hospitals Case Medical Center, 11100 \\ Euclid Avenue, Lakeside 3500, Cleveland, OH 44106-5028, USA \\ Email: Susan.Nedorost@UHhospitals.org
}

Published online: 30 July 2014

(C) Springer International Publishing AG 2014

Keywords Allergy - Contact dermatitis - Atopic dermatitis, patch test

\section{Opinion statement}

Atopic dermatitis $(A D)$ is a common chronic inflammatory skin disease resulting from a disrupted barrier function and an altered immune mechanism. Sometimes, it is difficult to distinguish between $A D$, irritant contact dermatitis (ICD), and allergic contact dermatitis (ACD) because they all present as eczematous dermatitis and may co-exist. Impaired barrier function in $A D$ predisposes to the development of both $A C D$ and ICD, as these three conditions share some mechanisms. The prevalence of ACD is at least as common in AD patients as in the general population; therefore, patients with chronic recalcitrant dermatitis should be evaluated for and considered for patch testing. Patch testing should also be undertaken in difficult-to-control or new-onset dermatitis in patients with $A D$, as avoiding the culprit allergen(s), along with barrier repair and infection control, will lead to improvement of symptoms. There are some special considerations when patch testing in patients with AD. Patch testing should include a standard basic screen in addition to the components of the emollients and cleansers, topical antibiotics, and topical corticosteroids used by the patient. 'Hypoallergenic' products without fragrances and strongly allergenic preservatives are commonly recommended in order to minimize the risk of irritation and sensitization via inflamed skin; however, AD patients are often sensitized to weakly potent allergens. Therefore, testing for an extended series of weak allergens used in personal care products and medicaments is critically important. Atopy patch testing (APT) may be also performed as an additional test to detect food and aeroallergen triggers of dermatitis in $A D$, if clinically indicated. This type of testing is also indicated in patients with a history of perioral eczema and no history of immediate hypersensitivity symptoms to the specific food tested. Aeroallergen APT is specifically indicated in seasonal dermatitis on exposed skin.

\section{Introduction}

Atopic dermatitis (AD) is a chronic inflammatory skin disease commonly found in children with a prevalence rate of $6 \%$ in the USA [1]. A majority of cases have onset within the first 5 years of life. AD 
clears in about $40 \%$ of children and may persist in some adults [2].

Symptoms of AD result from multiple factors, including genetic predisposition, impaired barrier functions, and altered immune mechanisms against environmental triggers and microbes.

Irritant contact dermatitis (ICD) and allergic contact dermatitis (ACD) often co-exist with $\mathrm{AD}$, and the three share some common cellular mechanisms $[3,4 \bullet]$. Loss-offunction mutations of the filaggrin gene have been associated with $\mathrm{AD}$. Filaggrin function has an effect on moisture retention in the epidermis [5]. Previous studies have shown that $\mathrm{AD}$ patients with filaggrin gene mutations have an increased risk of developing chronic ICD [6]. A disrupted barrier in $\mathrm{AD}$ leads to increased antigen penetration, activation of innate immunity, increased access of surface antigens to dendritic cells, and activation of the T helper (Th) 2 immune response. Th2 and Th17 predominate in patients with acute $\mathrm{AD}$ and Th22 and Th1 in patients with chronic disease [4•]. In addition, danger signals produced in inflamed skin of $\mathrm{AD}$ or ICD predispose to sensitization [7]. $\mathrm{AD}$ patients have baseline danger signal production from barrier impairment that predisposes to sensitization of weak allergens (Kohli N, Nedorost S, unpublished data).

The presence of IgE receptor molecules on epidermal Langerhans cells from patients with AD [8] may explain the higher possibility of cutaneous sensitization to large molecules such as proteins in patients with $\mathrm{AD}$. When an allergen is captured by an IgE molecule, it can then bind to the IgE receptor on an antigen presenting cell and be presented for immune recognition. Antigen presentation results in a $\mathrm{T}$ cell-mediated allergen-specific immune response that is responsible for the subsequent eczematous reaction [9].

\section{Prevalence of allergic contact dermatitis (ACD) in atopic dermatitis (AD)}

There are multiple factors that may affect the association between AD and contact sensitization, e.g., environmental exposures, diagnostic factors, and specific allergens considered [10•, 11-13].

In early studies, contact sensitivity to dinitrochlorobenzene (DNCB) in patients with $\mathrm{AD}$ was less than in control subjects [14] and severity of $\mathrm{AD}$ was inversely associated with contact sensitization. DNCB sensitization was positive in $33 \%$ of severe, $95 \%$ of moderate, and $100 \%$ of mild $\mathrm{AD}$ patients [15]. However, patients with severe AD do not have severe disease at all times, and therefore they may become sensitized during periods of milder disease activity. We speculate that severe $\mathrm{AD}$ in the setting of exposure to a strong sensitizer such as DNCB, which functions as its own irritant, may negatively affect dermal dendritic cell function. It has long been known that other noxious conditions such as ultraviolet $B$ will cause migration of dendritic cells away from the epidermis where antigens enter the skin and induce immune tolerance [16].

\section{Contact sensitization and the atopic march}

Patients with $\mathrm{AD}$ tend to develop IgE sensitization to proteins such as pollen, foods, and animal dander. Even though the antigen may penetrate the skin to an equal degree in atopic and non-atopic patients, AD patients are more likely to exhibit a Th2-skewed antigen response $[17 \bullet \bullet]$. AD patients with barrier dysfunction are also at increased risk of become sensitized to large protein allergens via skin contact, such as plant-derived 
proteins. These constituents may also be added to personal care products because of their moisturizing properties; oat and wheat, for example, may be used as ingredients and the risks of sensitization may need to be considered [18]. Type I and type IV reactions from skin contact (and systemic route in some cases) can occur [19-21].

\section{Identification of contact allergens in AD allows management by modification of lifestyle and diet}

Standard allergens included on most patch test screening series are as follows:

- Metals: AD patients with frequent episodes of hand eczema show a strong association between filaggrin gene mutations and contact sensitization to metals including, but not limited to, nickel [22-24].

- Medicaments: The most common allergens include neomycin [25], antiseptics, and antimicrobials such as chloroxylenol [26].

- Corticosteroids: Positive patch tests to tixocortol-21-pivalate were found in $2.5 \%$ of $\mathrm{AD}$ patients. Risk factors associated with positive patch test reactivity were $\mathrm{AD}$ severity, onset before the age of 6 months, IgE-mediated sensitization [27], and weak sensitizers (Kohli N, Nedorost S, unpublished data). In another study, $48 \%$ of AD patients had an allergic reaction to at least one allergen, and $12.8 \%$ had an allergic reaction to at least one corticosteroid [28].

- Fragrance: A German study found no difference in the prevalence of fragrance sensitization in patients with or without AD [29]. However, other studies (specifically in the last decade) have found a higher prevalence of fragrance sensitization in patients with $\mathrm{AD}$ than in controls [30-32].

- Preservatives: Recent data show increased risk of sensitization to some preservatives and surfactants in $\mathrm{AD}$ patients [33, 34]. We caution against the authors' interpretation of this data that patients with AD should avoid certain sensitizers while favoring others, because exposure and concentration was not controlled in these retrospective studies.

- Plant resins: Higher prevalence of sensitization to plant members of the Compositae family has been observed in patients with $\mathrm{AD}$ in some studies $[26,35,36]$. Paulsen and Andersen suggest that patients with $\mathrm{AD}$ may become sensitized to Compositae at any age, both occupationally and non-occupationally [36]. Several centers test children with seasonal flares on exposed skin to dandelion.

Atopy patch tests (APT) are performed by epicutaneous application of protein allergens, usually used to elicit standard IgE-dependent reactions tested by skin-prick test (SPT) [37], such as foods and aeroallergens. 
- Foods: AD flares by foods have been reported [38]. Some patients benefit from avoidance of milk, egg, soy, or birch-related foods [39] identified by APT.

- Aeroallergens: AD patients develop flexural contact dermatitis in later childhood and some of them have exacerbation of $\mathrm{AD}$ after contact with or inhalation of aeroallergens, e.g., house dust mites, pollen, or animal dander, and improve after avoidance [40].

The sensitivity and specificity varies widely depending on allergens [41]. SPT, specific IgE (sIgE), and APT have been studied for detecting food and aeroallergen sensitization in AD patients; they have been considered as diagnostic tools, but their predictive capacity varies by allergen and also for detection of early or late-phase reactions [42]. SPT and sIgE detect immediate-type hypersensitivity, whereas APT may have a role in detection of delayed-type hypersensitivity reactions. Further research to standardize and validate APT is needed.

\section{Testing indications}

\section{Patch test indications}

- New pattern of dermatitis

- Dermatitis fails to respond to previously successful treatment

- Suspicion of occupational dermatitis

\section{Patch test preliminaries}

- Test when back is dermatitis free in the test area, and patient is preferably off both topical and systemic corticosteroids

- Treat superficial/secondary infections with antibiotics before testing

- Treat post-pubertal patients with head and neck/textile pattern dermatitis with systemic anti-yeast antibiotics before testing

\section{Patch test selection}

- Allergens to be patched should be customized for each patient by detailed history and physical examination. A majority of $\mathrm{AD}$ cases are children and sometimes space on the patient's back is limited

- Test personal care products and medicament components in all patients

- Test occupational contactants when indicated

- Test foods in children with a history of perioral involvement

- Test aeroallergens in patients with seasonal flares of exposed skin

- Test for systemic contact dermatitis, e.g., to propylene glycol or benzoic acid, in patients with recurrent dermatitis in the same areas 


\section{Factors that may affect patch test results}

- Irritant, angry-back reactions may occur. Delayed reading (at 96 and $120 \mathrm{~h}$ ) helps reduce false positive interpretations due to irritant reactions. AD patients may have a follicular response from occlusion with patch-test tapes. Bacterial proliferation under patch-test occlusion may occur and cause flares of $\mathrm{AD}$. We recommend pre-treating adult $\mathrm{AD}$ patients who have active disease with systemic anti-staphylococcal and anti-yeast antibiotics before patch testing.

\section{Education on allergen avoidance and treatment of ACD in AD}

\section{Education on allergen avoidance}

- Patients must be educated on alternative medicaments and personal care products in order to completely avoid identified allergens. If the allergen may be ingested and systemic contact dermatitis is suspected, then instruction should be given in dietary avoidance. In our Midwestern US population, this is most common with food allergens such as oats, and food additives such as propylene glycol and benzoic acid.

- Education and counseling to the patient, parents of afflicted children, and primary health care providers is one the most important factors in helping with treatment of $\mathrm{ACD}$ in $\mathrm{AD}$. Counseling is the most timeconsuming, and most critical, aspect of treating AD.

- Education of other health care providers involved in the patient's care is also essential. These providers must know to avoid prescribing medications for the dermatitis that may contain the patient's allergens. Topical therapies with corticosteroids, emollients, calcineurin inhibitors, and oral corticosteroids may contain identified allergens as active or inactive ingredients such as propylene glycol and benzoic acid.

\section{Treatment of ACD in AD}

- The treatment approach should include the need to optimize skin barrier functions and prevent and treat bacteria and infection. In postpubertal patients sensitized to yeast [43], efforts to maintain skin decolonization of yeast are also important.

- As historical treatment of AD is primarily based on basic skin care and long-term topical treatments with emollients and topical corticosteroids, focusing on the potential for past or future sensitization to anything that comes into contact with the skin, including topical formulations used to treat $\mathrm{AD}$, is critical. Introducing new products on inflamed skin should be avoided as this may increase the risk of sensitization. 
- Use bleach baths to reduce commensal bacteria in the biofilm. Follow bleach baths with low $\mathrm{pH}$ emollients to promote filaggrin function and improve skin barrier [44-46].

\section{Prevention of ACD}

- Avoid contact of food with skin until tolerance is well-established. Even mild $\mathrm{AD}$ promotes sensitization; try to avoid skin contact with new foods until established feeding allows mucosal contact to induce tolerance. Prohibiting self-feeding in infancy might help with this.

- Avoid self-feeding in infants with hand eczema.

\section{Pediatric considerations}

- The overall prevalence of relevant positive patch tests reported by the NACDG (North American Contact Dermatitis Group) does not differ between children (51.2\%) and adults (54.1\%). Among positive reactions, $34.0 \%$ of children had $\mathrm{AD}$ compared with $11.2 \%$ of adults [47]. The data suggest that $\mathrm{AD}$ is a more important risk factor for $\mathrm{ACD}$ in children than in adults. However, this could be because AD is more common in children [47].

- Children with AD demonstrate contact allergy to components of personal care products and clothing such as nickel, neomycin, disperse dyes, and Myroxylon pereirae [25].

\section{Compliance with Ethics Guidelines}

\section{Conflict of Interest}

Suwimon Pootongkam declares that she has no conflict of interest.

Susan Nedorost declares that she has no conflict of interest.

Human and Animal Rights and Informed Consent

This article does not contain any studies with human or animal subjects performed by any of the authors.

\section{References and Recommended Reading}

Papers of particular interest, published recently, have been highlighted as:

- Of importance

- Of major importance

1. Hanifin JM, Reed ML. Eczema Prevalence and Impact Working Group. A population-based survey of eczema prevalence in the United States. Dermatitis. 2007;18:82-91.

2. Rudikoff D, Lebwohl M. Atopic dermatitis. Lancet. 1998;351:1715-21.

3. De Benedetto A, Kubo A, Beck LA. Skin barrier disruption: a requirement for allergen sensitization? J Invest Dermatol. 2012;132:949-63.

4. Gittler JK, Krueger JG, Guttman-Yassky E. Atopic dermatitis results in intrinsic barrier and immune abnormalities: implications for contact dermatitis. J Allergy Clin Immunol. 2013;131:300-13.

This article gives a nice summary and discusses cellular pathways associated with increased skin inflammation in $\mathrm{ACD}, \mathrm{ICD}$, and $\mathrm{AD}$. It also presents mechanisms that might contribute to the increased rate of ICD and ACD in patients with $\mathrm{AD}$. 
5. O'Regan GM, Sandilands A, McLean WH, et al. Filaggrin in atopic dermatitis. J Allergy Clin Immunol. 2009;124:R2-6.

6. de Jongh CM, Khrenova L, Verberk MM, et al. Loss-offunction polymorphisms in the filaggrin gene are associated with an increased susceptibility to chronic irritant dermatitis: a case-control study. Br J Dermatol. 2008;159:621-7.

7. Matzinger P. Tolerance, danger, and the extended family. Annu Rev Immunol. 1994;12:991-1045.

8. Bruijnzeel-Koomen CAFM, Van Wichen DF, Toonstra J, et al. The presence of IgE molecules on epidermal Langerhans cells from patients with atopic dermatitis. Arch Dermatol Res. 1986;278:199-205.

9. Wistokat-Wülfing A, Schmidt P, Darsow U, et al. Atopy patch test reactions are associated with T-lymphocyte mediated allergen-specific immune responses in atopic dermatitis. Clin Exp Allergy. 1999;29:513-21.

10. Thyssen JP, McFadden JP, Kimber I. The multiple factors affecting the association between atopic dermatitis and contact sensitization. Allergy. 2014;69:28-36.

This review article provides an overview of the multiple factors that affect the association between $\mathrm{AD}$ and contact sensitization. This will help readers to better understand these two conditions and will be useful for the management of ACD in $\mathrm{AD}$.

11. de Groot AC. The frequency of contact allergy in atopic patients with dermatitis. Contact Dermatitis. 1990;22:273-7.

12. Bieber T. Atopic dermatitis. N Engl J Med. 2008;358:1483-94

13. Eyerich K, Novak N. Immunology of atopic eczema: overcoming the Th1/Th2 paradigm. Allergy. 2013;68:974-82.

14. Rees J, Friedmann PS, Matthews JN. Contact sensitivity to dinitrochlorobenzene is impaired in atopic subjects. Controversy revisited. Arch Dermatol. 1990;126:1173-5.

15. Uehara M, Sawai T. A longitudinal study of contact sensitivity in patients with atopic dermatitis. Arch Dermatol. 1989;125:366-8.

16. Alcalay J, Kripke ML. Antigen-presenting activity of draining lymph node cells from mice painted with a contact allergen during ultraviolet carcinogenesis. J Immunol. 1991;146:1717-21.

18.• Newell L, Polak ME, Perera J, et al. Sensitization via healthy skin programs Th2 responses in individuals with atopic dermatitis. J Invest Dermatol. 2013;133:2372-80.

Demonstrates that potent sensitizers do create memory T cell responses in atopic dermatitis patients.

18. Pootongkam S, Nedorost S. Oat and wheat as contact allergens in personal care products. Dermatitis. 2013;24:291-5.

19. Vansina S, Debilde D, Morren MA, et al. Sensitizing oat extracts in cosmetic creams: is there an alternative? Contact Dermatitis. 2010;63:169-71.

20. Sanchez-Pérez J, Sanz T, García-Díez A. Allergic contact dermatitis from hydrolyzed wheat protein in cosmetic cream. Contact Dermatitis. 2000;42:360.
21. Livideanu C, Giordano- Labadie F, Paul C. Contact dermatitis to hydrolyzed wheat protein. Contact Dermatitis. 2007;57:283-4.

22. Thyssen JP, Linneberg A, Ross-Hansen K, et al. Filaggrin mutations are strongly associated with contact sensitization in individuals with dermatitis. Contact Dermatitis. 2013;68:273-6.

23. Dotterud LK, Falk ES. Metal allergy in north Norwegian schoolchildren and its relationship with ear piercing and atopy. Contact Dermatitis. 1994;31:308-13.

24. Malajian D, Belsito DV. Cutaneous delayed-type hypersensitivity in patients with atopic dermatitis. J Am Acad Dermatol. 2013;69:232-7.

25. Jacob SE, Yang A, Herro E, et al. Contact allergens in a pediatric population: association with atopic dermatitis and comparison with other North American referral centers. J Clin Aesthet Dermatol. 2010;3:29-35.

26. Nedorost ST, Babineau D. Patch testing in atopic dermatitis. Dermatitis. 2010;21:251-4.

27. Mailhol C, Lauwers-Cances V, Rancé F, et al. Prevalence and risk factors for allergic contact dermatitis to topical treatment in atopic dermatitis: a study in 641 children. Allergy. 2009;64:801-6.

28. Kot M, Bogaczewicz J, Krecisz B, et al. Contact hypersensitivity to haptens of the European standard series and corticosteroid series in the population of adolescents and adults with atopic dermatitis. Dermatitis. 2014;25:72-6.

29. Schnuch A, Lessmann $\mathrm{H}$, et al. Contact allergy to fragrances: frequencies of sensitization from 1996 to 2002. Results of the IVDK. Contact Dermatitis. 2004;50:65-76.

30. Rystedt I. Contact sensitivity in adults with atopic dermatitis in childhood. Contact Dermatitis. 1985;13:1-8.

31. Heine G, Schnuch A, Uter W, et al. Type-IV sensitization profile of individuals with atopic eczema: results from the Information Network of Departments of Dermatology (IVDK) and the German Contact Dermatitis Research Group (DKG). Allergy. 2006;61:6116.

32. Thyssen JP, Linneberg A, Engkilde K, et al. Contact sensitization to common haptens is associated with atopic dermatitis: new insight. Br J Dermatol. 2012; 166:1255-61.

33. Shaughnessy CN, Malajian D, Belsito DV. Cutaneous delayed-type hypersensitivity in patients with atopic dermatitis: reactivity to topical preservatives. J Am Acad Dermatol. 2014;70:102-7.

34. Shaughnessy CN, Malajian D, Belsito DV. Cutaneous delayed-type hypersensitivity in patients with atopic dermatitis: reactivity to surfactants. J Am Acad Dermatol. 2014;70:704-8.

35. Belloni Fortina A, Romano I, Peserico A. Contact sensitization to Compositae mix in children. J Am Acad Dermatol. 2005;53:877-80.

36. Paulsen E, Andersen KE. Sensitization patterns in Compositae-allergic patients with current or past atopic dermatitis. Contact Dermatitis. 2013;68:27785. 
37. Turjanmaa K, Darsow U, Niggemann B, et al. EAACI/ GA2LEN position paper: present status of the atopy patch test. Allergy. 2006;61:1377-84.

38. Niggemann B, Reibel S, Wahn U. The atopy patch test (APT)- a useful tool for the diagnosis of food allergy in children with atopic dermatitis. Allergy. 2000;55:281-5.

39. Werfel T, Reekers R, Busche M, et al. Evidence for a birch pollen-specific cutaneous T-cell response in foodresponsive atopic dermatitis. Int Arch Allergy Immunol. 1999;118:230-1.

40. Clark RA, Adinoff AD. The relationship between positive aeroallergen patch test reactions and aeroallergen exacerbations of atopic dermatitis. Clin Immunol Immunopathol. 1989;53:S132-40.

41. Lipozencić J, Wolf R. The diagnostic value of atopy patch testing and prick testing in atopic dermatitis: facts and controversies. Clin Dermatol. 2010;28:38-44.

42. Darsow U, Laifaoui J, Kerschenlohr K, et al. The prevalence of positive reactions in the atopy patch test with aeroallergens and food allergens in subjects with atopic eczema: a European multicenter study. Allergy. 2004;59:1318-25.
43. Ramirez de Knott HM, McCormick TS, Kalka K, et al. Cutaneous hypersensitivity to Malassezia sympodialis and dust mite in adult atopic dermatitis with a textile pattern. Contact Dermatitis. 2006;54:92-9.

44. Hachem JP, Crumrine D, Fluhr J, et al. pH directly regulates epidermal permeability barrier homeostasis, and stratum corneum integrity/cohesion. J Invest Dermatol. 2003;121:345-53.

45. Sakai T, Hatano Y, Zhang W, Fujiwara S. Defective maintenance of $\mathrm{pH}$ of stratum corneum is correlated with preferential emergence and exacerbation of atopic-dermatitis-like dermatitis in flaky-tail mice. J Dermatol Sci. 2014;74:222-8.

46. Shi VY, Tran K, Lio PA. A comparison of physicochemical properties of a selection of modern moisturizers: hydrophilic index and $\mathrm{pH}$. J Drugs Dermatol. 2012;11:633-6.

47. Zug KA, McGinley-Smith D, Warshaw EM, et al. Contact allergy in children referred for patch testing: North American Contact Dermatitis Group data, 2001-2004. Arch Dermatol. 2008;144:1329-36. 